\title{
Metastatic breast carcinoma to mandible: A case report
}

\author{
Rajul Ranka ${ }^{1 *}$, Alka Hande ${ }^{1}$, Amol Gadbail $^{2}$, Minal Chaudhary ${ }^{1}$ and Shrenik Oswal ${ }^{3}$ \\ ${ }^{1}$ Department of Oral Pathology and Microbiology, Sharad Pawar Dental College and Hospital, Sawangi, Wardha, Maharashtra, India \\ ${ }^{2}$ Department of Dentistry, Indira Gandhi Government Medical College, Nagpur, India \\ ${ }^{3}$ Department of Oral and Maxillofacial Surgery, Sharad Pawar Dental College and Hospital, Sawangi, India
}

\begin{abstract}
Metastases in the oral cavity are rare and comprise approximately $1 \%$ of all oral malignancies. They usually involve the jaws but may also be found in the soft tissues and salivary glands. The most common metastatic malignancies in women are from primary cancers in the breasts. A case report of a 50-year-old female is presented here who underwent bilateral mastectomies two years back and now has presented with metastasis to mandible along with history of pulmonary metastasis, lymphedema of single arm and vesiculobullous lesion at surgical site. Diagnosis of metastasis was confirmed by histopathology giving picture of invasive ductal carcinoma. This case illustrates the importance of suspecting a metastatic lesion in the jaw. As these lesions are associated with a poor prognosis, early detection is of utmost importance.
\end{abstract}

\section{Introduction}

Metastasis, or metastatic disease, is the spread of cancer or disease from one organ or part to another not directly connected with it [1]. The spread of metastasis may occur via the blood or the lymphatic or through both routes. The metastases frequently occur in the lungs, liver, brain, and the bones [1]. In the diagnostic workup for malignant tumors of the oral and maxillofacial region, it is obligatory to consider the possibility of both primary tumors and metastatic tumors, although the latter has very low incidence compared to the rest of the skeletal metastasis [2]. Metastases in the oral cavity are rare and comprise approximately $1 \%$ of all oral malignancies. They usually involve the jaws but may also be found in the soft tissues and salivary glands [3].

In women, the commonest metastatic malignancies are from primary cancers in the breasts, kidneys, colorectal region, genital organs and thyroid glands, and in men they arise from the lungs, prostate, kidneys and colorectal region [4,5].

Breast cancer is a fatal tumor in women; accounting for $15 \%$ of deaths from cancer [6]. Distant metastases in breast cancer may spread to almost any region of the body. Typically, metastases to regional lymph nodes are observed in nearly one-third of patients with cancer in the breast, colon, uterus, oral cavity, and pharynx [7]. Metastatic tumors are most often located in jaw bones (85\%) within the oral cavity. The bone most commonly involved is the mandible (80-90\%), mainly at molar or retromolar sites followed by maxilla and soft tissues [8].

Metastases to the oral cavity sometimes present with pain, ulcers on soft tissues, paresthesia of the involved region, bleeding, loosening of teeth, trismus, pathological fracture of the affected bone or remain asymptomatic. Radiographic examination most commonly shows a radiolucent osteolytic lesion with ill-defined and irregular margins and rarely osteoblastic lesions or mixed radiolucent and radiopaque lesions may be seen [9].

We are presenting a case report of a 50-year-old female who was operated case of breast carcinoma and presented with metastasis to the mandible which was confirmed by histopathology giving a picture of invasive ductal carcinoma.

\section{Case report}

A fifty-year-old female patient reported in outpatient department (OPD) of Sharad Pawar Dental College, Datta Meghe Institute of Medical Sciences, Sawangi (M), Wardha with a chief complaint of nonhealing ulcer, pain and swelling in right side of lower jaw. The patient gave a history of paresthesia and numbness in the lower lip on right side.

Extra-orally the swelling was $4 \times 5 \mathrm{~cm}$ approximately, lobulated, hard in consistency and tender on palpation. Puckering was present on the right cheek region (Figure 1A). Intraorally, an ulcerative lesion with soft tissue fixation was seen on the posterior-most part of right buccal mucosa in mandibular second and third molar region. It was approximately $2 \times 1 \mathrm{~cm}$ in size, roughly oval in shape with ill-defined borders and irregular surface showing teeth indentation (Figure 1B). The lesion was hard in consistency and induration was present.

On palpation, right submandibular lymphadenopathy was present with a palpable lymph node of size $2 \times 2 \mathrm{~cm}$ approximately. The patient was thin built, anorexic and had pallor suggestive of cachexia. There was pitting lymphedema on her right arm (Figure 1C). The patient also presented with blisters along with peeling and hard scars of blisters on chest (Figure 1D).

\section{Past medical history}

She was an operated case of bilateral breast carcinoma with a diagnosis of infiltrating duct carcinoma grade II and had 3 metastatic axillary lymph nodes. A total of six cycles of chemotherapy and twenty-eight cycles of radiotherapy had been taken by the patient. A

${ }^{*}$ Correspondence to: Rajul Ranka, Department of Oral Pathology and Microbiology, Sharad Pawar Dental College and Hospital, Sawangi (M), Wardha, Maharashtra, India, Tel: +91-855-496-9491; 8830361176; E-mail: rajul. ranka@gmail.com

Key words: breast carcinoma, invasive ductal carcinoma, mandible, metastasis

Received: September 19, 2018; Accepted: October 12, 2018; Published: October 17,2018 
computed tomography $(\mathrm{CT})$ chest (plain) reported pulmonary nodule on right upper lobe suggestive of right pulmonary metastasis. An Ultrasonography (USG) was done which reported mild splenomegaly. An orthopantomogram (OPG) examination revealed irregular osteolytic lesions suggestive of bone destruction on right ramus of mandible, involving right condyle and coronoid region with multiple small radiolucencies and small radiopaque foci (Figure 2). A posteroanterior (PA) chest examination revealed radiopacity on right lung suggestive of metastatic pleural effusion (Figure 3).

Considering the past medical history, a provisional diagnosis of metastatic breast carcinoma to the right side of the mandible was made. Consent was taken from the patient for a minor surgical procedure. Under local anesthesia biopsy of the lesion was taken, histopathology of which revealed, glandular tissue showing targetoid pattern in which tumor cells were arranged in the form of ducts (Figure 4A), also tumor cells were dispersed in fibrous connective tissue stroma in the form of strands and cords and Indian file appearance was present (Figure $4 \mathrm{~B})$. The neoplastic cells were round to oval in shape showing nuclear and cellular pleomorphism with hyperchromatic nuclei and abundant eosinophilic cytoplasm. Also, perineural and muscle invasion was seen (Figure 4C and 4D). Features were suggestive of invasive ductal carcinoma concluding metastatic breast carcinoma to the mandible.

To confirm the glandular tissue component and hyaluronic acid content in the tissue, alcian blue stain was performed which stained the connective tissue suggesting high hyaluronic acid content (Figure 5A).

Immunohistochemistry (IHC) was performed and tissue stained strongly positive for ER (Estrogen Receptor) (Figure 5B), PR

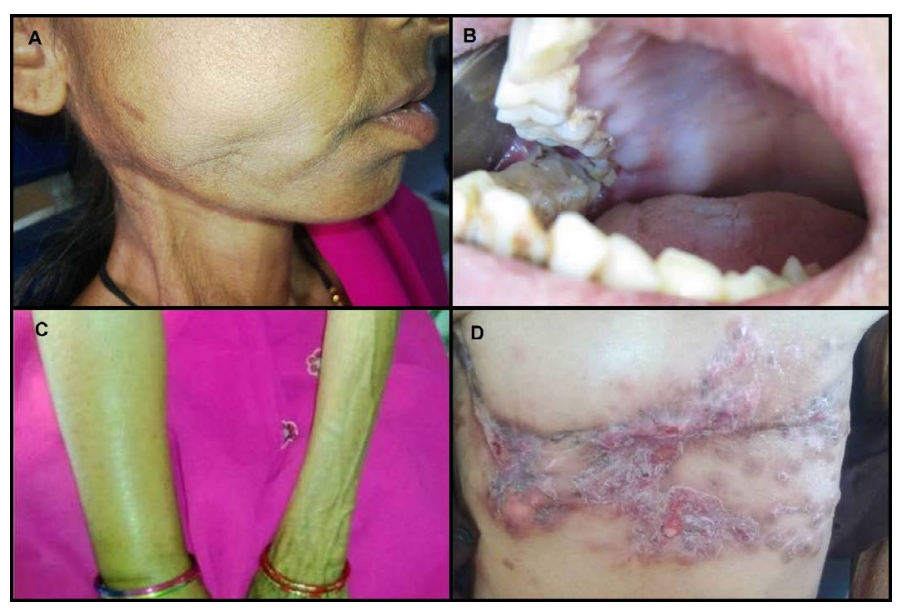

Figure 1. 1A- Puckering of cheek on right side; 1B- Intraoral growth with rough surface; 1C- Pitting lymphedema of right arm; 1D- Blisters and scars on chest wall

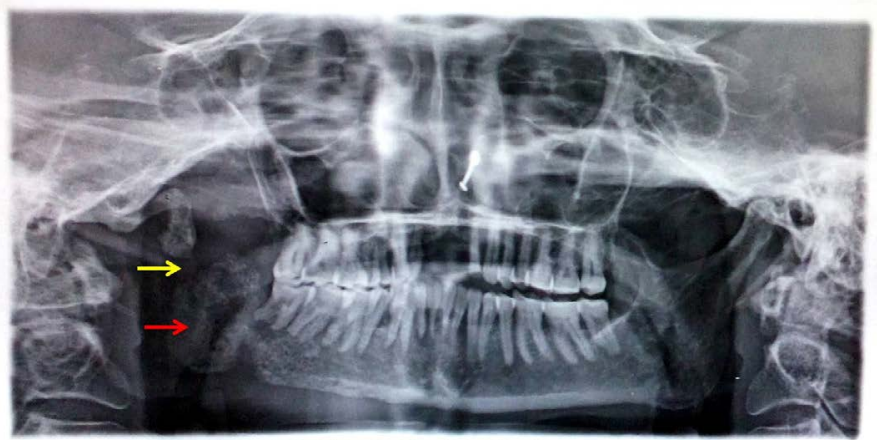

Figure 2. Orthopantomogram showing destruction of right mandibular ramus and condyle (yellow arrow); radiolucent and radiopaque foci (red arrow)

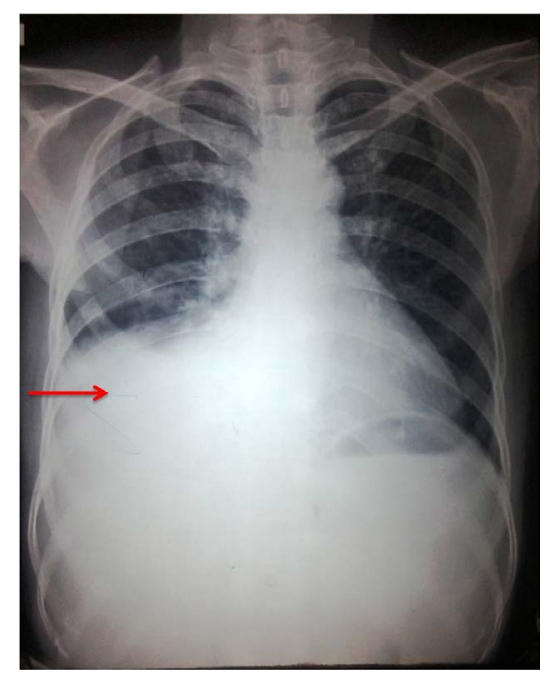

Figure 3. PA chest radiograph showing radiopacity on right lung suggestive of metastatic pleural effusion

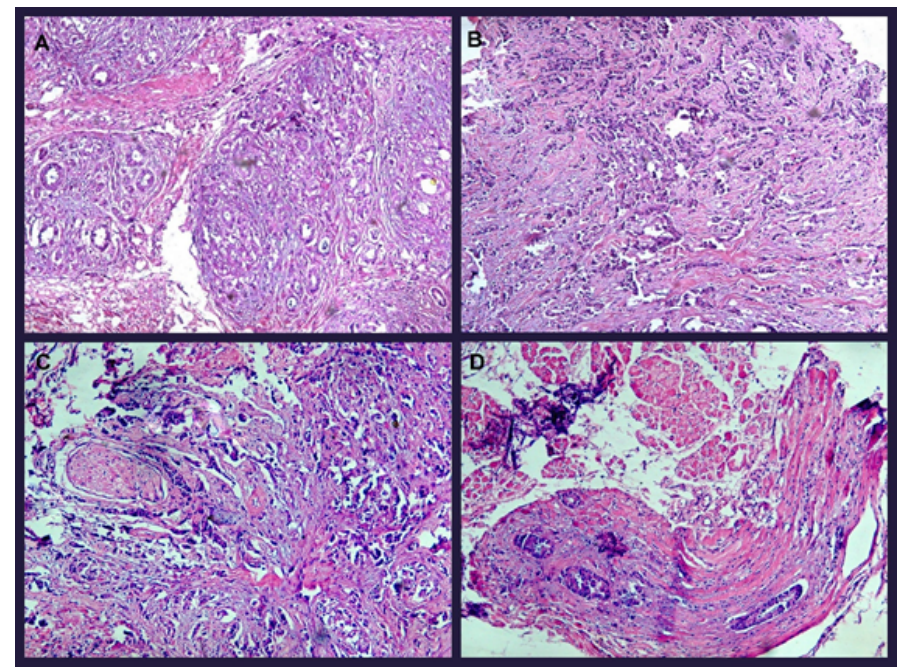

Figure 4. 4A- Targetoid pattern seen with tumor cells arranged in small ducts $(\mathrm{H} \& \mathrm{E}, 100 \mathrm{x})$ 4B- Tumor cells arranged in strands, cords and Indian file appearance is also seen (H\&E,100x); 4C- Tumor cells in close proximity to nerve suggestive of perineural invasion (H\&E,100x); 4D- Tumor cells invaded into the muscle tissue suggestive of muscle invasion (H\&E,100x)

(Progesterone Receptor) (Figure 5C) and HER2 (Human Epidermal Growth Factor Receptor) (Figure 5D).

The patient has then advised Positron emission tomography (PET) scan and palliative care with chemo-radiotherapy. The patient died in a span of two months suggesting poor prognosis.

\section{Discussion}

Theoretically, every extragnathic primary malignancy has the capability to metastasize to the oral cavity. Metastasis to the bone occurs more frequently than in the soft tissues [10]. The most frequent primary sites of malignancy with the potential to metastasize in the mandible are, in decreasing order, the breast (31\%), lung (18\%), kidney (15\%), thyroid, prostate and colon (6\%), stomach and skin (5\%), testicle (3\%), bladder, liver, uterus, and ovary (1\%) [11].

Metastatic lesions occur more often in the posterior region of the mandible, which is an area that retains its normal hematopoietic 


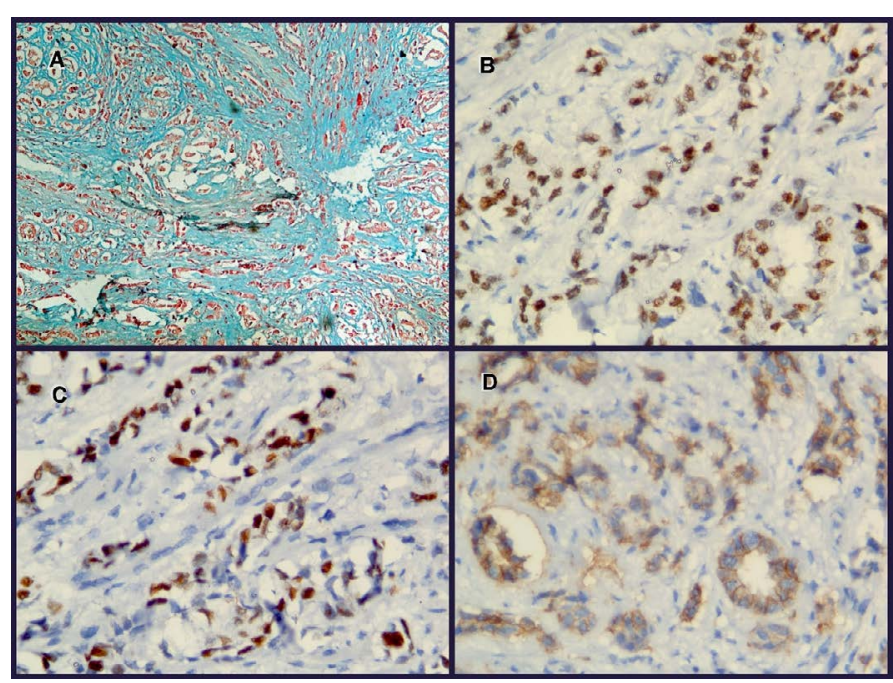

Figure 5. 5A- Alcian blue positive in connective tissue stroma of tumor (Alcian blue stain, 100x); 5B- Tumor cells positive for Estrogen receptor (ER) (IHC,100x); 5C- Tumor cells positive for Progesterone receptor (PR) (IHC,100x); 5D- Tumor cells positive for Human epidermal growth factor receptor (HER-2) (IHC,100x)

function. This possibly explains why the maxilla, although rich in spongy bone tissue, is a less common site for metastasis [11,12].

The most common symptom suggestive of metastatic disease is paresthesia of lower lip and chin. Paresthesia is described in the literature as mental nerve neuropathy or numb chin syndrome (NCS) [13].

Whenever the normal drainage pattern in the lymph nodes is disturbed or damaged, severe swelling of the arm may occur. The swelling that is caused by an abnormal accumulation of high content of fluid is called lymphedema. It may occur immediately following surgery, or months or years later. Women who have had many lymph nodes removed and women who have had radiation therapy have a higher risk of developing lymphedema [10]. This patient had pitting lymphedema of the right arm, swelling of which regresses on its own during the night and exacerbates during the daytime.

Local radiotherapy on surgical sites can cause redness, blistering and peeling of skin [14]. The patient presented with blisters on complete chest wall with scarring and peeling of skin.

Metastases of breast cancers identified to cause osteolytic lesions secrete biological mediators along with interleukin IL-11, IL-8, and IL-6, parathyroid hormone-related protein (PTHrP), that induce osteoclastmediated bone resorption through activation of the RANK/RANKL/ OPG signaling pathway. These mediators upregulate the expression of RANKL and down-regulate the expression of L(OPG) by osteoblasts and other stromal cells, as a consequence of promoting osteoclast differentiation and activation, culminating in bone resorption [15].

Hyaluronan is an anionic, nonsulfated glycosaminoglycan disseminated extensively throughout epithelial, connective, and neural tissues. Hyaluronan contributes conspicuously to cell proliferation and migration and is implicated in the progression of several malignant tumors. In several cancers, hyaluronan levels associate well with malignancy and poor prognosis. Hyaluronan, therefore, is frequently used as a tumor marker for breast and prostate cancer. It can also be used to supervise the progression of the disease. It stains positive for alcian blue in tissue sections [16]. In this case, the connective tissue component stained positive for alcian blue confirming the hyaluronic acid content.
The positive expression of ER \& PR suggests that the cancer cells growth is driven by the hormones estrogen and progesterone and will respond to hormone therapy. HER2 is a transmembrane tyrosine kinase receptor expressed by $15 \%-20 \%$ of invasive breast carcinomas. HER2 positive tumors grow more rapidly than HER2 negative tumors and respond to targeted therapy [17].

The prognosis is usually poor for the patients with metastatic lesion of the oral cavity, mainly because of the delay in the detection of the lesions. The survival time for patients with oral metastatic tumors is six to seven months on average, with about $70 \%$ of patients dying within one year of diagnosis [9].

\section{Conclusion}

The present case illustrates the value of suspecting a metastatic lesion in the oral cavity. A dental specialist must obtain the patient's complete medical history and cautiously evaluate unusual clinical findings such as lip and chin paresthesias and radiographic findings to differentiate metastatic lesions from clinically similar entities. The definite diagnosis can only be given by histopathologic examination. If histopathological, distant metastasis in the oral and maxillofacial region or elsewhere in the body of breast cancer patient is diagnosed, a bone scintigram or PET-scan has to be made to identify other tumor locations and to evaluate therapeutic options. For a patient with multiple metastases only palliative care with chemo-radiotherapy can be given improving the prognosis of the patient. As these lesions are associated with a poor prognosis, early detection is of utmost importance.

\section{References}

1. Klein CA (2008) Cancer. The metastasis cascade. Science 321: 1785-1787. [Crossref]

2. MEYER I, SHKLAR G (1965) Malignant tumors metastatic to mouth and jaws. Oral Surg Oral Med Oral Pathol 20: 350-362. [Crossref]

3. Hirshberg A, Buchner A (1995) Metastatic tumours to the oral region. An overview. Eur J Cancer B Oral Oncol 31B: 355-360. [Crossref]

4. D'Silva NJ, Summerlin DJ, Cordell KG, Abdelsayed RA, Tomich CE, et al. (2006) Metastatic tumors in the jaws: a retrospective study of 114 cases. J Am Dent Assoc 137: 1667-1672. [Crossref]

5. Dib LL, Soares AL, Sandoval RL, Nannmark U (2007) Breast metastasis around denta implants: a case report. Clin Implant Dent Relat Res 9: 112-115.

6. Greenlee RT, Hill-Harmon MB, Murray T, Thun M (2001) Cancer statistics, 2001. CA Cancer J Clin 51: 15-36. [Crossref]

7. der Waal RI, Buter J, van der Waal I (2003) Oral metastases: report of 24 cases. Br J Oral Maxillofac Surg 41: 3-6. [Crossref] van

8. Friedrich RE, Abadi M (2010) Distant metastases and malignant cellular neoplasms encountered in the oral and maxillofacial region: analysis of 92 patients treated at a single institution. Anticancer Res 30: 1843-1848. [Crossref]

9. Poulias E, Melakopoulos I, Tosios K (2011) Metastatic breast carcinoma in the mandible presenting as a periodontal abscess: a case report. J Med Case Rep 5: 265 [Crossref]

10. Ertas U, Yalcin E, Erdogan F (2010) Invasive Ductal Carcinoma with Multiple Metastases to Facial and Cranial Bones: A Case Report. Eur J Dent 4: 334-337. [Crossref]

11. Rocha WC, Curado MP, Vencio EF, Celestino Caixeta WB (200) Endometria carcinoma metastatic to the mandible: A case report. J Oral Maxillofac Surg 58: $914-$ 916. [Crossref]

12. Hashimoto N, Kurihara K, Yamasaki H, Ohba S, Sakai H, et al. (1987) Pathologica characteristics of metastatic carcinoma in the human mandible. J Oral Pathol 16: 362367. [Crossref]

13. Lesnick JA, Zallen RD (1999) Numb chin syndrome secondary to metastatic breas disease. J Colo Dent Assoc 78: 11-14. [Crossref] 
14. Stout NL, Pfalzer LA, Springer B, Levy E, McGarvey CL, et al. (2012) Breast cancerrelated lymphedema: comparingdirect costs of a prospective surveillance model and a traditional model of care. Phys Ther 92: 152-163. [Crossref]

15. Feller L, Kramer B, Lemmer J (2011) A short account of metastatic bone disease. Cancer Cell Int 11: 24. [Crossref]
16. Stern R (2008) Hyaluronidases in cancer biology. Semin Cancer Biol 18: 275-280. [Crossref]

17. Onitilo AA, Engel JM, Greenlee RT, Mukesh BN (2009) Breast cancer subtypes based on ER/PR and Her2 expression: Comparison of clinicopathologic features and survival. Clin Med Res 7: 4-13. [Crossref]

Copyright: (C2018 Ranka R. This is an open-access article distributed under the terms of the Creative Commons Attribution License, which permits unrestricted use, distribution, and reproduction in any medium, provided the original author and source are credited. 